OPEN ACCESS

Edited by:

Lorenza Putignani,

Bambino Gesù Ospedale Pediatrico

(Istituto di Ricovero e Cura a Carattere

Scientifico), Italy

Reviewed by:

Max Maurin

Université Grenoble Alpes, France Brian Weinrick

Albert Einstein College of Medicine

USA

*Correspondence:

Siouxsie Wiles

s.wiles@auckland.ac.nz

Received: 14 November 2016 Accepted: 13 March 2017 Published: 28 March 2017

Citation:

Dalton JP, Uy B, Swift S and Wiles S (2017) A Novel Restraint Device for Injection of Galleria mellonella Larvae that Minimizes the Risk of Accidental Operator Needle Stick Injury.

Front. Cell. Infect. Microbiol. 7:99. doi: 10.3389/fcimb.2017.00099

\section{A Novel Restraint Device for Injection of Galleria mellonella Larvae that Minimizes the Risk of Accidental Operator Needle Stick Injury}

\author{
James P. Dalton ${ }^{1,2,3}$, Benedict Uy ${ }^{1,2}$, Simon Swift ${ }^{2}$ and Siouxsie Wiles ${ }^{1,2,3 *}$ \\ ${ }^{1}$ Bioluminescent Superbugs Lab, University of Auckland, Auckland, New Zealand, ${ }^{2}$ Department of Molecular Medicine and \\ Pathology, University of Auckland, Auckland, New Zealand, ${ }^{3}$ Maurice Wilkins Centre for Molecular Biodiscovery, Auckland, \\ New Zealand
}

Larvae of the insect Galleria mellonella are increasingly being used for studying pathogenic microbes and their virulence mechanisms, and as a rapid model for screening novel antimicrobial agents. The larvae (waxworms) are most frequently infected by injection of pathogenic organisms into the haemocoel through the insect's prolegs. The mostly widely used method for restraining the waxworms for injection is by grasping them between the operator's fingers, which puts the operator at risk of needle stick injury, an important consideration when working with highly pathogenic and/or drug-resistant microorganisms. While use of a stab proof glove can reduce this risk of injury, it does so at the loss of manual dexterity and speed, resulting in a more labor-intensive, and cumbersome assay. We describe a simple cost effective device (the so-called "Galleria Grabber") for restraining waxworms for injection that keeps the operator's fingers clear of the needle thus reducing the risk of injury.

Keywords: Galleria grabber, infectious diseases, Staphylococcus aureus, caterpillar, bacteria

\section{INTRODUCTION}

Larvae (waxworms) of the Greater wax moth Galleria melonella have become a widely used surrogate host for studying pathogenic microbes. In recent years, they have been used for studying virulence mechanisms, investigating differences between clinical isolates as well as for preliminary investigation of the efficacy of antimicrobial compounds, for a wide range of both Gram-positive and Gram-negative bacteria (Joyce and Gahan, 2010; McLaughlin et al., 2012; Ramarao et al., 2012; Loh et al., 2013; Thomas et al., 2013; Williamson et al., 2014; Adamson et al., 2015; Champion et al., 2016; Johnston et al., 2016; Moreira et al., 2016; Nale et al., 2016; Yang et al., 2016), fungi (Alcazar-Fuoli et al., 2015; Forastiero et al., 2015; Borman et al., 2016; de Lacorte Singulani et al., 2016; Frenkel et al., 2016; Gago et al., 2016; Santos et al., 2016), and viruses (Garzon et al., 1978; Buyukguzel et al., 2007; Özkan and Coutts, 2015). The use of waxworms as a model host has many advantages. The waxworms themselves are cheap and easy to obtain from commercial insect suppliers, and can be housed in large numbers to allow for greater study sizes at low cost. Waxworms possess an innate immune system that contains many analogous functions to that seen in humans, including phagocytosis and the production of antimicrobial peptides and reactive oxygen and nitrogen species (Wojda, 2016). Unlike other non-mammalian model organisms, 
such as Caenorhabditis elegans, Danio rerio, and Drosophila melanogaster (Glavis-Bloom et al., 2012; Arvanitis et al., 2013; Panayidou et al., 2014; Lopez Hernandez et al., 2015), waxworms can be incubated at $37^{\circ} \mathrm{C}$ which allows for the study of clinically relevant human pathogens at a temperature that mimics the human host. Finally, as insects, G. mellonella are not currently subject to the same ethical restrictions that small mammalian models are, meaning there is a low barrier to entry for researchers wishing to move their studies into a model host.

Infection of waxworms is typically carried out on 5 th instar insects, when the waxworms are at their largest, typically around $2 \mathrm{~cm}$ in length and $100 \mathrm{mg}$ in weight. The most common method of infection is by injection into the haemocoel through the last proleg of the insect; methods for injection vary between laboratories. One method is to immobilize the needle itself and then place the waxworm onto the needle for injection. Another more favored method is to immobilize the waxworms between the operator's fingers (Fuchs et al., 2010) and place the needle into the insect's proleg, lifting the needle away from the operator with the insect attached before pushing the plunger on the syringe. Both of these injection techniques present a hazard to the researcher and can result in needle stick injury and possible infection.

A recent article highlighted the use of a stab-proof glove to reduce the chance of this type of injury while immobilizing the waxworms over a pipette tip fixed to some paper (Harding et al., 2013). We have tried this technique and found that, while safer for the operator, using a stab-proof glove reduces the efficiency of injection, from 3-4 to 1 infection per minute, resulting in a lower injection rate and a more labor-intensive assay. Because of this, we investigated the possibility of using a simple restraining device to hold waxworms in place for injection, in a way that removes the operator's hand from the vicinity of the needle, allowing for maximum mobility, and safety of the operator.

\section{MATERIALS AND METHODS}

\section{Preparation of Bacteria}

The Staphylococcus aureus isolate XEN36 (Francis et al., 2000) (Perkin Elmer) was grown overnight with shaking at $200 \mathrm{rpm}$ in Tryptic Soy broth (Oxoid) at $37^{\circ} \mathrm{C}$. Cells were washed twice in phosphate buffered saline (PBS) (Sigma-Aldrich) and then resuspended in PBS to an optical density at $600 \mathrm{~nm}\left(\mathrm{OD}_{600}\right)$ of 1 , equivalent to $\sim 5 \times 10^{9} \mathrm{CFU} \mathrm{ml}^{-1}$. Resuspended cultures were serially diluted and plated onto Tryptic Soy agar (Oxoid) to retrospectively determine the bacterial counts used for injection. Inoculation doses were drawn into $1 \mathrm{ml}$ ultra-fine (29 gauge) needle insulin syringes (BD, Wellington) for injection into the waxworms. Groups of waxworms were injected with $20 \mu \mathrm{l}$ of either phosphate-buffered saline (PBS) or $\sim 5 \times 10^{7}, 5 \times 10^{8}$, or $5 \times 10^{9} \mathrm{CFU} \mathrm{ml}^{-1}$ S. aureus XEN36.

\section{Selection, Infection, and Monitoring of G. mellonella Waxworms}

Fifth instar waxworms were selected based on consistency in size and split into eight groups of 12 . Four groups were injected with either PBS or doses of $10^{6}-10^{8}$ CFU S. aureus XEN36 using the most common technique of grasping the waxworms between the operator's thumb and index finger and injecting into the waxworm's last proleg. The remaining four groups were injected with either PBS or doses of $10^{6}-10^{8}$ CFU S. aureus XEN36 using the newly described restraining device (which we have dubbed the "Galleria Grabber"), which comprises a $12 \times 9 \mathrm{~cm}$ kitchen sponge and a large bulldog clip $(\sim 50 \mathrm{~cm})$ (Figure 1A). To comfortably restrain the waxworms, the sponge was folded in half and secured using the bulldog clip (Figure 1B). The open ends of the folded sponge were peeled back and held in place (Figure 1C). Next, a waxworm was placed within the sponge and held in place while the open end of the sponge was released (Figure 1D). Once the waxworm was securely held in place, the insulin syringe was inserted into the haemocoel via the insect's last proleg (Figure 1E). Once the needle was in place the waxworm was released from the restraining device (Figure 1F). If the needle is correctly placed, the waxworm remains attached to the needle of the syringe. Once the needle had been securely
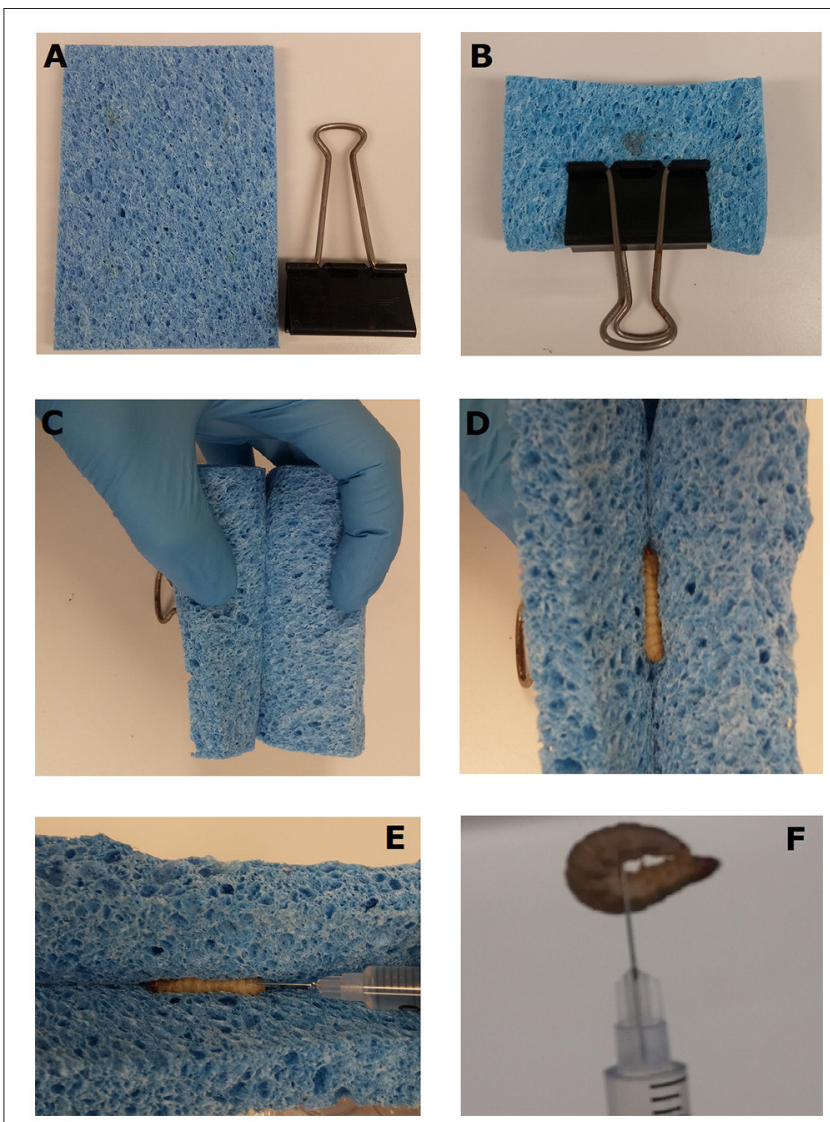

FIGURE 1 | Injection of waxworms using a novel restraint device. The "Galleria Grabber" restraint device is comprised of a $15 \mathrm{~mm}$ thick sponge and bulldog clip (A). The sponge is folded in half lengthways and secured within a bull dog clip with the open end facing outwards (B). The open ends of the folded sponge are peeled back and held in place (C). The waxworm to be injected is placed within the sponge and held in place while the open end of the sponge is released. The closing of the sponge secures the waxworm in place for injection (E). Once the needle is placed, the syringe is lifted with the waxworm in place and the plunger is pushed to inject the desired inoculum (F) 


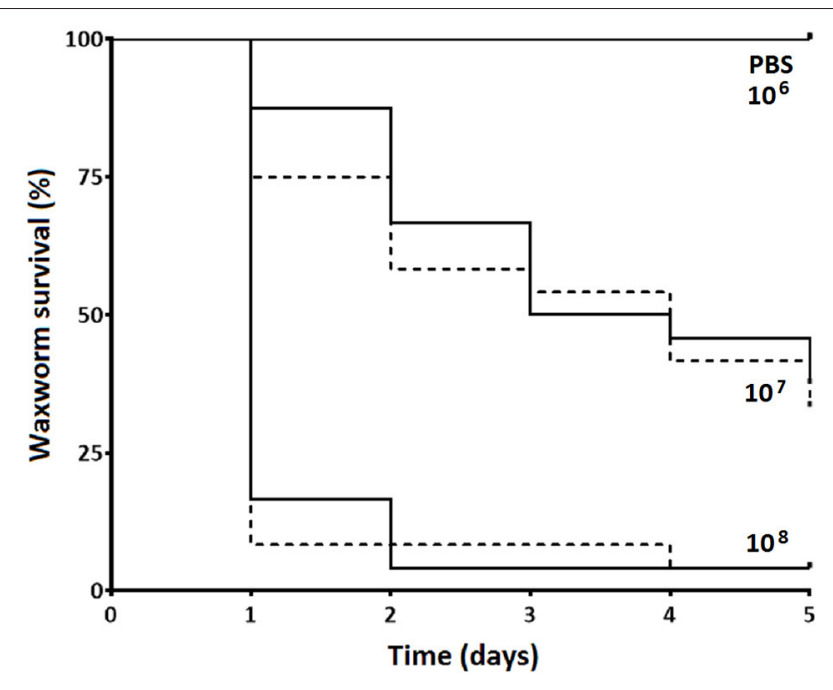

FIGURE 2 | Waxworms ( $n=12$ per group) were infected with varying concentrations of $S$. aureus XEN36 or phosphate-buffered saline (PBS) by injection into the haemocoel via the last proleg while restrained either between the thumb and index finger of the operator (solid lines), or using the "Galleria Grabber" restraint device (dashed lines), and survival measured over 5 days.

inserted into the waxworm, the insect was removed from the restraining device and the plunger of the syringe pushed down to inject the desired inoculum.

Once injected, waxworms were housed in individual wells of 24 well-tissue culture dishes (Nunc) with the lids taped down to ensure against escape. These dishes were placed inside a secondary container to ensure containment. Waxworm mortality was monitored over 5 days.

\section{RESULTS AND DISCUSSION}

We observed no differences in the infection dynamics between the groups of waxworms injected with $S$. aureus XEN36 after

\section{REFERENCES}

Adamson, D. H., Krikstopaityte, V., and Coote, P. J. (2015). Enhanced efficacy of putative efflux pump inhibitor/antibiotic combination treatments versus MDR strains of Pseudomonas aeruginosa in a Galleria mellonella in vivo infection model. J. Antimicrob. Chemother. 70, 2271-2278. doi: 10.1093/jac/dkv111

Alcazar-Fuoli, L., Buitrago, M., Gomez-Lopez, A., and Mellado, E. (2015). An alternative host model of a mixed fungal infection by azole susceptible and resistant Aspergillus spp strains. Virulence 6, 376-384. doi: 10.1080/21505594.2015.1025192

Arvanitis, M., Glavis-Bloom, J., and Mylonakis, E. (2013). Invertebrate models of fungal infection. Biochim. Biophys. Acta 1832, 1378-1383. doi: 10.1016/j.bbadis. 2013.03.008

Borman, A. M., Szekely, A., and Johnson, E. M. (2016). Comparative pathogenicity of United Kingdom isolates of the emerging pathogen Candidaauris and other key pathogenic Candida species. MSphere 1:e0018916. doi: $10.1128 / \mathrm{mSphere.00189-16}$ restraint using the novel "Galleria Grabber" device described compared to restraint by holding the waxworms between the operator's thumb and index finger. For both restraint techniques, we observed no mortality from the waxworms injected with PBS (Figure 2). In contrast, the majority of waxworms injected with $\sim 10^{8}$ CFU S. aureus XEN36 died within $24 \mathrm{~h}$ (Figure 2). We observed a dose dependent mortality for waxworms injected with S. aureus XEN36, with $66 \%$ of waxworms injected with $\sim 10^{5}$ CFU succumbing to infection (Figure 2). No mortality was seen after injection with $10^{6}$ CFU S. aureus XEN36 (Figure 2).

The "Galleria Grabber" allows for easy injection of a large number of waxworms ( $\sim 3$ per minute), while greatly reducing the opportunity for the operator to suffer a needle stick injury. With the increasing popularity of waxworms as a model host for studies involving dangerous human pathogens (Champion et al., 2016), including clinical and/or drug-resistant isolates, protecting researchers from accidental laboratory infection is of great importance. While the use of a stab-resistant glove addresses this issue, it does compromise the speed at which waxworms can be injected. With this new restraint method, we were also able to inject smaller waxworms with ease. Most importantly, the new methodology described removes the operator's hand from the vicinity of needles loaded with pathogenic/drug-resistant microbes, allowing for maximum mobility and safety of the operator without compromising the speed of the assay.

\section{AUTHOR CONTRIBUTIONS}

JD, Conceived and designed the experiments; JD, BU, Performed the experiments; JD, SW, Analyzed the data; SS, Contributed reagents; JD, SW, Wrote the manuscript; JD, SW, Prepared the figures; JD, BU, SS, SW, Reviewed drafts of the paper.

\section{FUNDING}

This work was supported by a University of Auckland new staff grant to SW (9802 3707601).

Büyükgüzela, E. Tunazb, H., Stanley, D., and Büyükgüzela, K. (2007). Eicosanoids mediate Galleria mellonella cellular immune response to viral infection. $J$. Insect. Physiol. 53, 99-105. doi: 10.1016/j.jinsphys.2006.10.012

Champion, O. L., Wagley, S., and Titball, R. W. (2016). Galleria mellonella as a model host for microbiological and toxin research. Virulence 7, 840-845. doi: 10.1080/21505594.2016.1203486

de Lacorte Singulani, J., Scorzoni, L., de Paula, E. S. A. C., Fusco-Almeida, A. M., and Mendes-Giannini, M. J. (2016). Evaluation of the efficacy of antifungal drugs against Paracoccidioides brasiliensis and Paracoccidioides lutzii in a Galleria mellonella model. Int. J. Antimicrob. Agents 48, 292-297. doi: 10.1016/j.ijantimicag.2016.05.012

Forastiero, A., Bernal-Martínez, L., Mellado, E., Cendejas, E., and Gomez-Lopez, A. (2015). In vivo efficacy of voriconazole and posaconazole therapy in a novel invertebrate model of Aspergillus fumigatus infection. Int. J. Antimicrob. Agents 46, 511-517. doi: 10.1016/j.ijantimicag.2015.07.007

Francis, K. P., Yu, J., Bellinger-Kawahara, C., Joh, D., Hawkinson, M. J., Xiao, G., et al. (2000). Monitoring bioluminescent Staphylococcus aureus infections in 
living mice using a novel luxABCDE construct. Infect. Immun. 68, 3594-3600. doi: 10.1128/IAI.68.6.3594-3600.2000

Frenkel, M., Mandelblat, M., Alastruey-Izquierdo, A., Mendlovic, S., Semis R., and Segal, E. (2016). Pathogenicity of Candida albicans isolates from bloodstream and mucosal candidiasis assessed in mice and Galleria mellonella. J. Mycol. Med. 26, 1-8. doi: 10.1016/j.mycmed.2015.12.006

Fuchs, B. B., O’Brien, E., Khoury, J. B., and Mylonakis, E. (2010). Methods for using Galleria mellonella as a model host to study fungal pathogenesis. Virulence 1, 475-482. doi: 10.4161/viru.1.6.12985

Gago, S., Serrano, C., Alastruey-Izquierdo, A., Cuesta, I., Martín-Mazuelos, E., Aller, A., et al. (2016). Molecular identification, antifungal resistance and virulence of Cryptococcus neoformans and Cryptococcus deneoformans isolated in Seville, Spain. Mycoses 60, 40-50. doi: 10.1111/myc.12543

Garzon, S., Charpentier, G., and Kurstak, E. (1978). Morphogenesis of the nodamura virus in the larbae of the lepidopteran Galleria mellonella (L.). Arch. Virol. 56, 61-76. doi: 10.1007/BF01317283

Glavis-Bloom, J., Muhammed, M., and Mylonakis, E. (2012). Of model hosts and man: using Caenorhabditis elegans, Drosophila melanogaster and Galleria mellonella as model hosts for infectious disease research. Adv. Exp. Med. Biol. 710, 11-17. doi: 10.1007/978-1-4419-5638-5_2

Harding, C. R., Schroeder, G. N., Collins, J. W., and Frankel, G. (2013). Use of Galleria mellonella as a model organism to study Legionella pneumophila infection. J. Vis. Exp. e50964. doi: 10.3791/50964

Johnston, T., Hendricks, G. L., Shen, S., Chen, R. F., Kwon, B., Kelso, M. J., et al. (2016). Raf-kinase inhibitor GW5074 shows antibacterial activity against methicillin-resistant Staphylococcus aureus and potentiates the activity of gentamicin. Future Med. Chem. 8, 1941-1952. doi: 10.4155/fmc-2016-0104

Joyce, S. A., and Gahan, C. G. (2010). Molecular pathogenesis of Listeria monocytogenes in the alternative model host Galleria mellonella. Microbiology 156, 3456-3468. doi: 10.1099/mic.0.040782-0

Loh, J. M., Adenwalla, N., Wiles, S., and Proft, T. (2013). Galleria mellonella larvae as an infection model for group A streptococcus. Virulence 4, 419-428. doi: 10.4161/viru.24930

López Hernández, Y., Yero, D., Pinos-Rodríguez, J. M., and Gibert, I. (2015). Animals devoid of pulmonary system as infection models in the study of lung bacterial pathogens. Front. Microbiol. 6:38. doi: 10.3389/fmicb.2015.00038

McLaughlin, H. P., Xiao, Q., Rea, R. B., Pi, H., Casey, P. G., Darby, T., et al. (2012). A putative P-type ATPase required for virulence and resistance to haem toxicity in Listeria monocytogenes. PLoS ONE 7:e30928. doi: 10.1371/journal.pone.0030928

Moreira, A. S., Mil-Homens, D., Sousa, S. A., Coutinho, C. P., Pinto-deOliveira, A., Ramos, C. G., et al. (2016). Variation of Burkholderia cenocepacia virulence potential during cystic fibrosis chronic lung infection. Virulence doi: 10.1080/21505594.2016.1237334. [Epub ahead of print].
Nale, J. Y., Chutia, M., Carr, P., Hickenbotham, P. T., and Clokie, M. R. (2016). 'Get in Early'; Biofilm and Wax Moth (Galleria mellonella) models reveal new insights into the therapeutic potential of Clostridium difficile bacteriophages. Front. Microbiol. 7:1383. doi: 10.3389/fmicb.2016.01383

Özkan, S., and Coutts, R. H. (2015). Aspergillus fumigatus mycovirus causes mild hypervirulent effect on pathogenicity when tested on Galleria mellonella. Fungal Genet. Biol. 76, 20-26. doi: 10.1016/j.fgb.2015.01.003

Panayidou, S., Ioannidou, E., and Apidianakis, Y. (2014). Human pathogenic bacteria, fungi, and viruses in Drosophila: disease modeling, lessons, and shortcomings. Virulence 5, 253-269. doi: 10.4161/viru.27524

Ramarao, N., Nielsen-Leroux, C., and Lereclus, D. (2012). The insect Galleria mellonella as a powerful infection model to investigate bacterial pathogenesis. J. Vis. Exp. e4392. doi: 10.3791/4392

Santos, R., Costa, C., Mil-Homens, D., Romão, D., de Carvalho, C. C., Pais, P., et al. (2016). The multidrug resistance transporters CgTpol_1 and CgTpo1_2 play a role in virulence and biofilm formation in the human pathogen Candida glabrata. Cell. Microbiol. e12686. doi: 10.1111/cmi.12686. [Epub ahead of print].

Thomas, R. J., Hamblin, K. A., Armstrong, S. J., Müller, C. M., Bokori-Brown, M., Goldman, S., et al. (2013). Galleria mellonella as a model system to test the pharmacokinetics and efficacy of antibiotics against Burkholderia pseudomallei. Int. J. Antimicrob. Agents 41, 330-336. doi: 10.1016/j.ijantimicag.2012. 12.009

Williamson, D. A., Mills, G., Johnson, J. R., Porter, S., and Wiles, S. (2014). In vivo correlates of molecularly inferred virulence among extraintestinal pathogenic Escherichia coli (ExPEC) in the wax moth Galleria mellonella model system. Virulence 5, 388-393. doi: 10.4161/viru.27912

Wojda, I. (2016). Immunity of the greater wax moth Galleria mellonella. Insect Sci. doi: 10.1111/1744-7917.12325. [Epub ahead of print].

Yang, H., Chen, G., Hu, L., Liu, Y., Cheng, J., Ye, Y., et al. (2016). Enhanced efficacy of imipenem-colistin combination therapy against multiple-drugresistant Enterobacter cloacae: in vitro activity and a Galleria mellonella model. J. Microbiol. Immunol. Infect. doi: 10.1016/j.jmii.2016.01.003. [Epub ahead of print].

Conflict of Interest Statement: The authors declare that the research was conducted in the absence of any commercial or financial relationships that could be construed as a potential conflict of interest.

Copyright (c) 2017 Dalton, Uy, Swift and Wiles. This is an open-access article distributed under the terms of the Creative Commons Attribution License (CC BY). The use, distribution or reproduction in other forums is permitted, provided the original author(s) or licensor are credited and that the original publication in this journal is cited, in accordance with accepted academic practice. No use, distribution or reproduction is permitted which does not comply with these terms. 